\title{
Figures and boxes
}

\section{FIGURES}

2.1 Influence and incentive 19

2.2 Decision-making and knowledge 19

3.1 Illustrative representation of the event study method 33

3.2 Excess residuals by group of firms 42

6.1 Crisis communication lifecycle (adapted from Reynolds, 2002) 86

10.1 UNECE Model for Risk Management in Regulatory Systems

BOX

10.1 Contingency planning in standards 162 